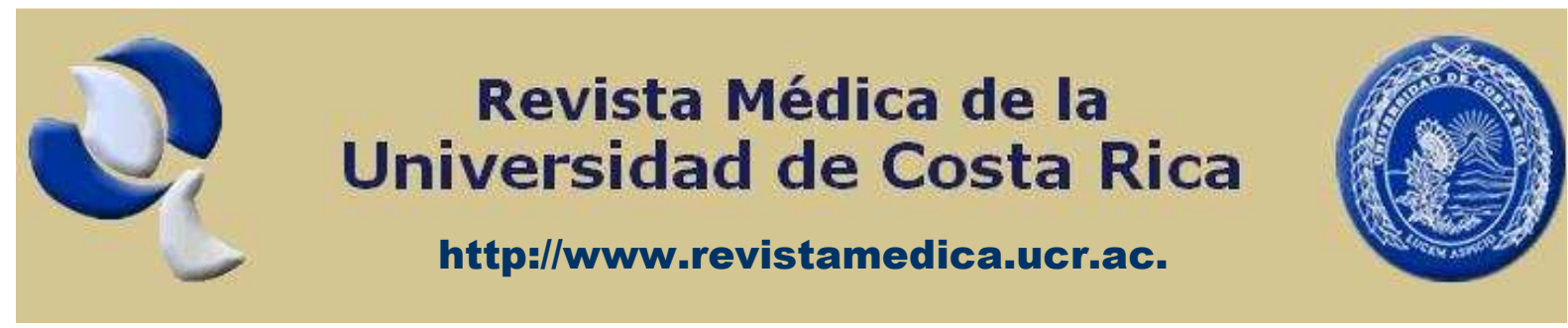

Personajes sobresalientes de la Medicina Nacional

\title{
EL DR. JUAN JARAMILLO ANTILLÓN; AUTOBIOGRAFÍA
}

\author{
Jaramillo Antillón, Juan
}

Profesor emérito de la Escuela de Medicina de la Universidad de Costa Rica. San Pedro, Costa Rica.

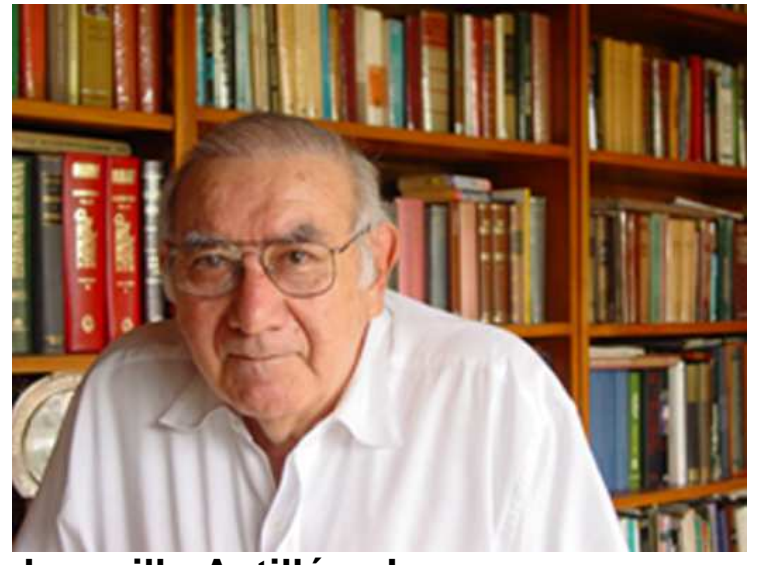

Jaramillo Antillón, Juan

Recibido: Diciembre 2007. Aceptado: Febrero 2008. Publicado: Marzo 2008

P: ¿Cuándo y dónde nació? R: En San José, barrio la Dolorosa 1935. P: ¿Sus padres tenían recursos? $\mathrm{R}$ : Mi padre Juan Jaramillo y mi madre Arabela Antillón, pertenecían a la clase trabajadora. Lo que más recuerdo de ellos es que al llegar la noche papá, después de un día de intenso trabajo, se dedicaba como contador a llevar los libros de varias compañías para ganarse una extra y mamá cosía vestidos de novia y hacia queques para vender. Con esa extra, me enviaron a estudiar al Colegio de Los Ángeles, toda la primaria y secundaria.

P: ¿Hermanos? R: Solo uno. Orlando, un brillante dermatólogo, ex jefe de Dermatología del Hospital México, Catedrático y Profesor Emérito de la UCR. P: ¿Cuándo se caso y cuántos hijos tuvo. R: Me case con Mabel Borges, cuando cumplí 21 años y ella 18, estaba en 4 año de la carrera de Medicina, este año cumplí 52 felices años de matrimonio y si algo logre en mi vida, se debió a su apoyo y cariño. Cuatro hijos Yasmín, Jefe de Psiquiatría 
del Hospital de Niños. Juan Carlos Administrador de Empresas y trabaja en el BNCR. Vanesa, abogada y quien me ha dado los únicos dos nietos que tengo de 15 y 17 años Diego y Nicole. Cynthia Publicista y Psicóloga quien vive feliz desde hace muchos años en San Francisco, USA con su marido James un Ingeniero norteamericano y otro hijo más para mí.

P: ¿Usted siempre deseo ser médico? $\mathrm{R}$ : No, cuando termine el bachillerato no tenía una idea clara de a que dedicarme, me gustaba la arquitectura pero no había carrera en $\mathrm{CR}$, dos amigos ticos que habían iniciado los estudios de medicina en México, me ofrecían ayuda para vivir en su apartamento con un gasto mínimo y entonces mis padres realizando un gran esfuerzo, me dieron la mitad de sus ingresos para que me fuera a estudiar a ese país, con ello se condenaban a no tener un carro propio, ni a tener una casa buena. Fue así como en 1953 fui a estudiar medicina ahí.

$P$ : ¿Fue un buen estudiante, le tomo gusto a la carrera? R: Los dos primeros años fueron muy duros y a ratos quería regresarme a Costa Rica, sin embargo logre superar mis temores que todo estudiante joven tiene y logre terminar la carrera en 1959 con una Mención Honorífica en la Universidad Nacional Autónoma de México D.F.

P: ¿Realizó internado y Servicio Social en México? R: Si, el internado que era el último año de la carrera fue básico para adquirir cierta experiencia, para luego ir al Servicio Social de 6 meses requisito para poder presentar el examen profesional. A quienes lo realizaban en lugares lejanos le pagaban 5 mil pesos mensuales. Este lo realice a $3.500 \mathrm{~km}$ de la Capital, en un pueblo San Javier, Sinaloa, en un Ejido del gobierno donde vivían 5 mil familias campesinas, mestizas o indias, a las cuales el Estado les había proporcionado tierras de cultivo (pequeñas parcelas) y ellos cultivaban ahí maní y maíz que era vendido a intermediarios que pagaban muy mal por los cultivos. Eran muy pobres y solamente tenían médico cada 6 meses al año. No había clínica, farmacia y menos hospital, el más cercano quedaba a $200 \mathrm{~km}$. En los alrededores vivían unas 20 mil personas. No había carretera para autos, solo un camino para camiones pesados y dentro del pueblo y sus alrededores andaba a caballo.

P: ¿Principales experiencias? $\mathrm{R}$ : La primera y más tremenda fue la soledad como persona y como médico que sentí al encontrarme tan aislado y sin recursos técnicos (no equipos, no medicinas, no farmacia) y ausencia de los maestros que me guiaran ante los problemas

de una población pobre que veía al médico como un dios capaz de resolver todas sus dolencias, algo que me deprimía pues sabía de mis limitaciones.

$\mathrm{P}$ : ¿Fue útil la experiencia? R: Fue fundamental para formarme un carácter ya que tuve que hacer frente y solo a numerosos problemas de obstetricia, medicina interna, traumatismo, heridas, picaduras de serpiente y alacrán, problemas de pediatría. El pueblo me dio una casa de un solo gran cuarto frente a una Iglesia que tenía 50 años de no funcionar. La casa no tenía lavatorio, baño y existía escusado de hueco en el patio. No había cañería ni agua potable, esta la traían diariamente

Revista electrónica publicada por el Departamento de Farmacología de la Escuela de Medicina de la Universidad de Costa Rica, 2060 San José, Costa Rica. ${ }^{\circledR}$ All rights 
de un río que pasaba cerca, se hervía y se usaba para preparar alimentos, tomar café etc., y en mi caso para esterilizar los instrumentos para hacer pequeñas operaciones. Cada 3 días me iba a bañar al igual que el resto del pueblo a una poza en el río más o menos a media hora a caballo.

$P$ : ¿Sus experiencias más traumáticas. $\mathrm{R}$ : Tuve dos problemas que me dieron mucha fama. El primero un niño muy pobre de 7 años con un absceso en el lado derecho del abdomen, al palparlo me di cuenta por el dolor al rebote y la masa que dolía era un absceso apendicular. Los padres dos inditos no podían llevarlo al hospital pues no tenían plata. Improvise una camilla, esterilice mis instrumentos, me ayudo la maestra del lugar como instrumentista y enfermera, y bajo anestesia local, apenas hice una incisión pequeña y el pus dreno ampliamente, felizmente sin tener que entrar a cavidad, la fístula que había dejado el absceso se cerro y el niño curo. Aquí me di cuenta que la suerte o Dios protege a los inocentes como el paciente y mi persona. El segundo caso muy traumático a 4 horas de caballo en la sierra, fue a atender una india de parto con tres días de labor, las comadronas ya sabían que el niño había muerto y no podía salir. La razón ellas mismas me la dieron, su cabeza era muy grande, la mujer ya no tenía fuerzas y estaba infectada. Se trataba de un hidrocéfalo, y tuve que proceder a una craneotrípsia o sea a destruir con un bisturí la cabeza del niño para sacar su cerebro y el líquido que contenía y poder entonces sacar fragmentos de los huesos del cráneo y el resto del cuerpo. Para mi asombro a los dos días la madre se levanto y se fue a bañar al río y volvió a cocinar para sus otros hijos y su esposo. La experiencia fue terrible, pero me decidió a que yo sería obstetra o cirujano.

P: ¿Usted cobraba algo por la consulta?

R: Cobraba "a quien podía pagar, 2 pesos por la consulta" si no podían con dinero, lo hacían con huevos, gallinas, conejos, carne de cerdo, frutas, etc. La amistad y comprensión que me dieron los vecinos en ese lugar me hicieron la vida más llevadera ahí y me humanizaron en el sentido de darme cuenta lo valiente que eran esas gentes para vivir y trabajar sin quejarse.

P: ¿Cuándo regreso a Costa Rica y en que fechas hizo el internado y el Servicio Social de aquí? R: En Septiembre de 1959, y trate de realizar el internado en el Hospital San Juan de Dios, el único autorizado para ello. Lamentablemente las 12 plazas de interno estaban ocupadas y 25 colegas recién graduados de México, Argentina, España, etc., estaban desde hacía meses trabajando gratuitamente (meritoriamente) como decía el Dr. Pena Chavarría director del hospital, quien contaba (abusando de los jóvenes médicos) con el doble de internos sin paga, ya que para entrar al internado había que hacer por meses cola como se dice. En visto de que estaba casado y con una niña, necesitaba un salario para vivir. Provisionalmente mis formidables suegros Abelardo Borges e Irma Muñoz, me daban casa y comida, por lo que en unión con otros compañeros fuimos a hablarle al Dr. José MI Quince (q.p.d), quien era en ese entonces Ministro de Salud, y estuvo anuente a darnos plaza de Servicio Social sin haber hecho internado. Me toco la Unidad Sanitaria de Limón. A partir del 1 de Noviembre, pero antes y

Revista electrónica publicada por el Departamento de Farmacología de la Escuela de Medicina de la Universidad de Costa Rica, 2060 San José, Costa Rica. ${ }^{\circledR}$ All rights reserved. 
durante 3 semanas asistimos a un excelente curso de medicina rural y comunitaria que brindaba el Dr. Romero (un distinguido medico salvadoreño exilado aquí) y que nos llevo cada día a diferentes unidades sanitarias rurales del país a ver cómo funcionaban y permanecíamos ahí todo el día. En Limón, no encontraba casa para mi familia debido a lo caro en la ciudad, felizmente el Dr. Domingo Argüello director del Hospital, me ofreció una excelente casa en la Zona de la Antigua Compañía Bananera que rodeaba al hospital. El pago por ella lo hacía mediante una guardia en el hospital un fin de semana completo (sábado y domingo) cada quince días y un día entre semana. Con ello pude traerme a mi señora e hija a Limón. Como no existía carretera y se viajaba por avión o en el tren el pachuco que duraba 8 horas de San José a Limón en la noche. Trabajaba 3 días en la Unidad Sanitaria atendiendo a los pobres no asegurados de la ciudad y viajaba a atender enfermos a dispensarios del Ministerio en Batán, Zent, Matina, Cahuita y Puerto Viejo, 2 veces a la semana.

P: ¿Usted considera la experiencia del Servicio Social importante, pues ya sabe que ahora prácticamente está eliminado, pues hay muy pocas plazas? $\mathrm{R}$ : La experiencia fue para mí fundamental para conocer no solo los problemas médicos de la zona, sino además, los problemas económicos y sociales que condicionan muchas enfermedades y para las cuales no tenía respuesta como médico. Por otro lado el trato con esa gente tan buena de esa provincia llena de necesidades me hizo conocer la forma de ser del costarricense en especial de los descendientes afrocaribeños de esa zona, sus costumbres, alimentación, alegrías y tristezas, experiencia inolvidable que me sirvió posteriormente en mi vida profesional, sobre todo porque deje muchos amigos y pacientes que años después me visitaban en San José.

P: ¿Cuándo hizo el internado? R: Lo inicié en septiembre de 1961 en el Hospital Calderón Guardia. Ahí tuve la oportunidad de hacer amistad con un grupo de excelentes médicos que marcarían mi vida en muchos sentidos. Los doctores Longino Soto Pacheco Jefe de Cirugía 1 del hospital, quien posteriormente se dedico a cirugía cardiovascular y realizo los primeros transplantes con buenos resultados de corazón en Centroamérica, y su Jefe de Clínica el Dr. Arnoldo Fernández Soto. Ambos fueron no solo mis maestros durante el internado y los dos primeros años del la Residencia en Cirugía, sino además mis amigos y protectores. Posteriormente llegó como Jefe de Sección el Dr. Manuel Aguilar

Bonilla (pocos años después seria vicepresidente de Costa Rica) y me toco estar los dos últimos años de la residencia en su Servicio. Su calidad humana y preparación terminaron de consolidar mi preparación en Cirugía General y además, me consiguió una beca para ir a hacer gastroenterología medico quirúrgica, y proctología, al Servicio de Gastroenterología del Centro Médico Nacional del Seguro Social de México en el Distrito Federal.

$\mathrm{P}$ : ¿Al regreso donde trabajó? $\mathrm{R}$ : Felizmente el Dr. Aguilar tenía cuatro horas de cirujano para las que concursé y gané, en la tardes daba consulta externa en la Clínica Central cuyo director era el Dr. Pedro Cuendis, y para redondearme un salario hacía visitas a

Revista electrónica publicada por el Departamento de Farmacología de la Escuela de Medicina de la Universidad de Costa Rica, 2060 San José, Costa Rica. ${ }^{\circledR}$ All rights reserved. 
domicilio de 5 pm a 9 pm diariamente. En el hospital establecí una afortunada amistad con los doctores Guido Miranda, uno de los pioneros de la modernización del Seguro Social y en ese entonces Jefe de Medicina Interna del Hospital. El Jefe de Urología, Dr.

Fernando Coto Chacón, y Montalto, el Dr. Carlos Prada, ginecólogo del hospital y quienes me participaron de sus inquietudes sobre la seguridad social del país y de hecho me condujeron a dedicar parte de mi tiempo a estudiarla y colaborar con ellos para universalizar el Seguro Social de Costa Rica.

P: ¿Pero usted se fue posteriormente al Hospital México? R: Si, al abrirse el hospital México en el año de 1969, los doctores Aguilar Bonilla y Guido Miranda seleccionaron a un grupo de médicos y cirujanos del hospital para que se trasladaran del Calderón al México y entre ellos iba yo. El Hospital Calderón Guardia fue prácticamente desmantelado, no solo de sus recursos humanos sino también de equipos, y se trataría de hacer ahí un Hospital Geriátrico. Con la ampliación de la cobertura del Seguro Social, fallaron a muy corto plazo los pronósticos y ya para mediados del año 1972, a solo tres años de estar funcionando el México no daba abasto y hacían falta muchas camas y las operaciones se estaban atrasando meses. En el México trabaje como Jefe de Clínica de Cirugía General en el Servicio del Dr. Fernández Soto. A mediados de 1972, las autoridades de la Caja, entre ellos el Lic. Rodrigo Fournier Gerente de la misma y mis amigos el Dr. Manuel Aguilar y Guido Miranda me pidieron que me regresara al Hospital Calderón Guardia, en calidad de Jefe de Cirugía, en unión de un grupo de jóvenes colegas y amigos de otras especialidades a organizar los diferentes servicios y pasar de 200 camas a 450 en el menor tiempo posible. Para ello se nos ofrecía darnos "todo lo que necesitáramos" entre ellos un nuevo edificio y que la Escuela de Medicina de la UCR estableciera Cátedras paralelas ahí.

$\mathrm{P}:$ ¿Usted se regresó? $\mathrm{R}: \mathrm{Si}$, decidimos dejar todo lo que teníamos de ventajas para hacer medicina en el Hospital México e ir a levantar a ese "Cementerio de Elefantes" como le decían al Calderón. Fue tal la pasión y el esfuerzo que todos pusimos que una vez que logramos se hiciera la Torre Médica terminada en 1980, y contando con las 450 camas y 17 salas de operaciones, ya para esa época y pese a tener menos camas que el San Juan de Dios y el México teníamos más internamientos y atendíamos más emergencias que esos hospitales y proporcionalmente a las camas de cirugía, realizábamos más operaciones mensuales que los otros hospitales, esto se mantuvo así por lo menos hasta 1998, cuando me retire siendo jefe de la Sección de Cirugía por muchos años y por 20 años director de la Cátedra de cirugía de la Escuela de Medicina de la UCR.

P: ¿En el campo profesional que puestos desempeñó usted? Desde interno a asistente especialista de cirugía general, Jefe de Clínica y Servicio de Cirugía General y finalmente Jefe de toda la Sección de Cirugía en los hospitales México y Calderón Guardia.

P: ¿Qué especialidades tenía? R: Especialista en Cirugía General y

Revista electrónica publicada por el Departamento de Farmacología de la Escuela de Medicina de la Universidad de Costa Rica, 2060 San José, Costa Rica. ® All rights 
Especialista en Proctología (Enfermedades del Colon y Recto). Además, por varios años fui Coordinador y Miembro de la Comisión Nacional de Hospitales de la CCSS., Asesor Médico de la Junta Directiva de la CCSS y de dos Presidentes Ejecutivos, así como de tres Ministros de Salud.

P: ¿En el campo de la docencia que puestos desempeño usted? R: Trabaje para la Escuela de Medicina de la UCR desde 1966 a 1998. Fui Instructor encargado de Cátedra, Profesor Adjunto y Asociado. Profesor Titular y finalmente Director y Coordinador de Cátedra y Catedrático de Medicina. Fui Miembro Asesor del Decano de la Escuela de Medicina varios años. Miembro de la Comisión para elaborar el Nuevo Currículo. Como Miembro del Programa de Post Grado de la UCR y la CCSS, elaboré el primer programa de Post-Grado de Cirugía. Finalmente fui declarado Profesor Emérito de la Universidad de Costa Rica. Por cierto, mientras estuvo vigente que los estudiantes al graduarse escogieran un Padrino de la Generación, fui padrino cinco veces, al parecer el profesor que más número de veces se le ha hecho ese reconocimiento en la Escuela de Medicina de la UCR.

P: ¿Usted fue Ministro de Salud, en que período y cuales considera sus principales logros?. R: Lo fui en el período de 1982-1986, época en la que el país pasó la peor crisis económica del último siglo. Pese a ello y a que en los 4 años los presupuestos no crecieron, fuimos capaces de sostener y aún mejorar los índices de salud del país. Creamos el Sector Salud con la rectoría del Ministro y por primera vez se inició la
Integración y Coordinación de servicios de salud y médicos entre la CCSS y el Ministerio de Salud, creándose así el inicio de un sistema preventivo asistencial para llegar a cubrir a toda la población del país sin distingo de condición económica y disminuir costos. Con ello ampliamos la llamada Atención Primaria de la Salud y se incorporaron los indigentes a los servicios médicos de la Caja.

Creamos también las Primeras Juntas de Salud y Seguridad Social en los 80 Cantones del país, para darle participación comunitaria a la población en la toma de decisiones en ese campo, proceso que vino a finalizarse en el 2002.

Obtuvimos del Congreso un presupuesto mediante una Ley con ayuda de dos diputadas, para crear el programa CEN-CINAI, atención integral del niño pequeño menor de 6 años pobre en nutrición y educación, y de las madres embarazadas de escasos recursos, actualmente en vigencia, por esa época sólo existían programas de nutrición en los CEN totalmente desfinanciados.

Creamos el primer Formulario Terapéutico Nacional, con el fin de fijar la cantidad y calidad de medicamentos que la CCSS ofrecería a sus pacientes.

Creamos el Consejo Nacional de Cáncer y el primer programa de Fluorización de la Sal, con ayuda de la Fundación Kellog. Obligamos a que las empresas de cigarrillos y licor pusieran en sus productos. "El fumar o beber licor es dañino para su salud".

P: ¿Premios y Honores recibidos? R: Numerosos, entre ellos: Mención > Honorífica en mi graduación de Médico. Miembro de Honor en diversos Congresos Médicos y de Salud, algunos

Revista electrónica publicada por el Departamento de Farmacología de la Escuela de Medicina de la Universidad de Costa Rica, 2060 San José, Costa Rica. ${ }^{\circledR}$ All rights reserved. 
con mi nombre. Reconocimientos de las Juntas Directivas de la CCSS por mis servicios en diferentes ocasiones. De la Universidad de Costa Rica, del Gobierno, etc. Miembro de Honor de la Asociación de Salud Pública y de la de Cirugía, Declarado Notable en el campo de la Medicina por el Gobierno de Costa Rica 1994. Presidente de la Unión Médica y Primer Presidente de la Academia Nacional de Medicina y Miembro de Honor de la misma, Así como del Colegio de Médicos y Cirujanos.

Premio Nacional de Literatura en la rama de ensayo, Aquileo Echeverría. Premio por Distinguishe Service Award (Premio IHMEC) de las Escuelas de Medicina de los Estados Unidos y Canadá, por mi contribución a mejorar la calidad de la enseñanza de la medicina en Costa Rica y Latinoamérica.

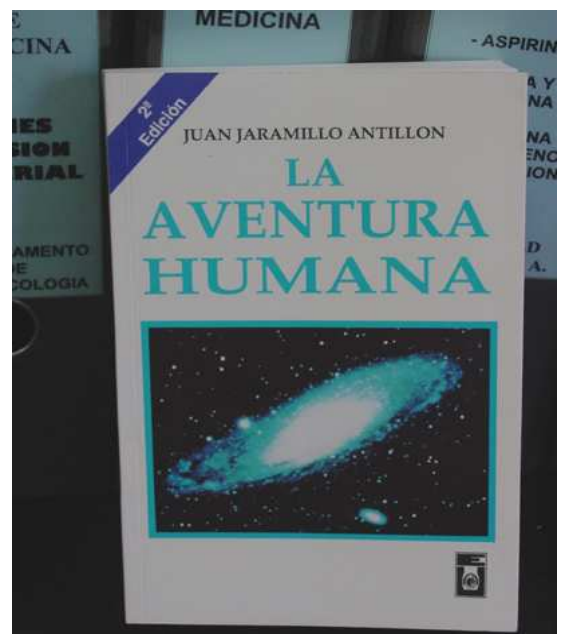

Catedrático de Medicina y Profesor Emérito de la UCR. Nombrado Par académico junto con dos expertos extranjeros por el SINAES, para evaluar y acreditar por primera vez una escuela de medicina (la de la UCR). Premio Ancora en Ciencia del periódico la Nación.
P: ¿Miembro de Asociaciones 0 Academias del país o del extranjero? R: Miembro de 20, entre ellas de Fellow Internacional College of Surgeons. Fellow Internacional Collegue of Angiology, $\mathrm{De}$ las Sociedades Mexicanas de Gastroenterología y Proctología, de la Academia Nacional de Ciencias de Nueva York y de la Asociación Americana para el Avance de la Ciencia en Estados Unidos.

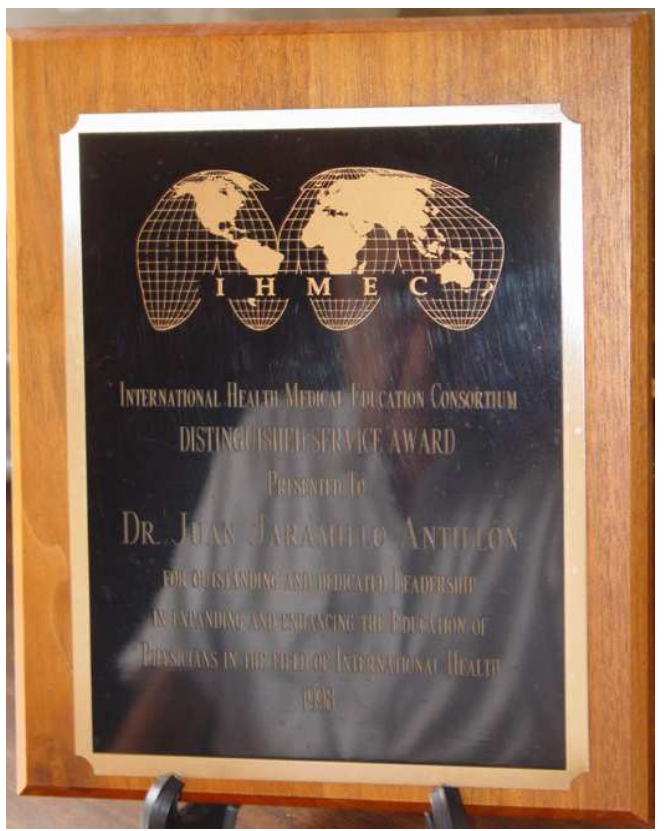

P: ¿Libros, folletos, publicaciones. Se dice que usted es el autor más prolífico que tiene el país en el campo de la medicina, la salud y la seguridad social? R: No lo sé, sin embargo, he publicado: 27 libros. 36 ensayos o folletos. 80 artículos en revistas médicas o de salud nacionales o del exterior y 205 artículos por periódicos.

P: ¿Participación como expositor o conferencista en Reuniones, Congresos Médicos, de Salud o de Seguridad Social en el país o en el exterior?

Revista electrónica publicada por el Departamento de Farmacología de la Escuela de Medicina de la Universidad de Costa Rica, 2060 San José, Costa Rica. ${ }^{\circledR}$ All rights reserved. 
Participe en esa modalidad en 132 de ellos.

$\mathrm{P}$ : ¿Estudios en Investigación? R: Realice varios en Investigación Clínica en compañía de algunos compañeros o solo, en el campo de la Integración de los Servicios de salud en CR. Otro sobre las enfermedades tiroideas del país, sobre cáncer gástrico, y otros cánceres y sobre la calidad de la enseñanza de la medicina en Costa Rica. Producto final de eso fueron dos libros publicados en 1985 por el Ministerio de Salud sobre Organización y

Funcionamiento del Sector Salud y la Atención Médica en el País. Además, publiqué en 1978 con el Dr. Eric Mora los resultados de nuestros estudios sobre ENFERMEDADES DEL TIROIDES $Y$ PARATIROIDES. Otra personal sobre CANCER GASTRICO EN COSTA RICA 1988, y un libro de Dos tomos que dirigí y fui el principal colaborador sobre EL CANCER en Costa Rica, aquí colaboraron 20 de los principales cancerólogos del país en las diferentes ramas. Todos con la Editorial de la UCR.

P: ¿Cirugías realizadas? Varios miles durante mi vida, pero en especial me enorgullezco de haber ayudado a decenas de jóvenes cirujanos residentes a realizar miles de operaciones bajo $\mathrm{mi}$ dirección y cuidado. Creo que un buen cirujano que no ayuda y forma una escuela, no deja ninguna herencia en el campo de la medicina.

$\mathrm{P}$ : ¿Entretenimientos? $\mathrm{R}$ : La lectura desde niño sobre todo de historia, ciencias, biografías, filosofía, etc. Y la música clásica en especial la ópera que lamentablemente dejé de apreciar pues me quedé sordo a los 30 años.
P: Para finalizar, deseo preguntarle, ¿bajo qué premisas ha desarrollado su trabajo a través de su vida?. R: Aprendí de mis padres "que no hay comida gratis" y que para ganarse un salario no importa el puesto o la posición que uno ocupe, hay que trabajar y sobre todo trabajar bien y honestamente, ya qué el trabajo lo dignifica a uno y es entonces respetado.

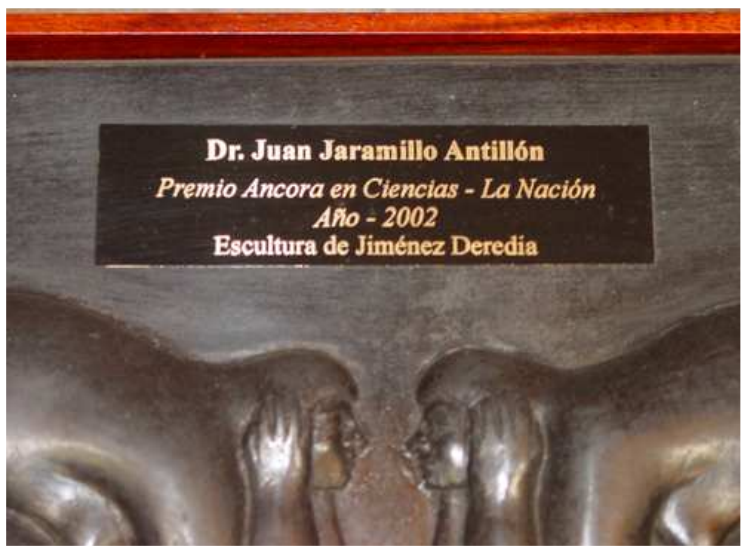

Los médicos deben ampliar su horizonte cultural con conocimientos en otros campos fuera de la medicina para lograr una mejor formación cultural y ver al paciente como un ser humano y no como un simple enfermo. De esta manera he trasmitido a mis alumnos la idea de que para curar, es importante no solo saber de medicina, sino comprender al ser humano como un todo, donde el respeto a la persona forma parte del arte de la medicina y la valora.

Además, debemos ser humildes, ya que vuelvo a repetir, el mejor médico no es el que no se equivoca sino el que se equivoca menos, ya que ha aprendido más de los errores que de los éxitos y somos apenas ayudantes de la naturaleza para curar.

Revista electrónica publicada por el Departamento de Farmacología de la Escuela de Medicina de la Universidad de Costa Rica, 2060 San José, Costa Rica. ${ }^{\circledR}$ All rights reserved. 
Hoy aceptamos que las ciencias médicas y la protección de la salud, forman posiblemente la parte más importante de apoyo a la sociedad al proteger "el capital" humano, el insumo más importante que tiene una nación. Por eso se hace necesario humanizar estos campos, para darle un contenido más benevolente y espiritual a nuestras acciones. Así como darle un contenido más social al descubrimiento científico y ejercer cada vez un mejor control sobre las investigaciones y la aplicación de sus resultados en los humanos.

Por esa razón siempre insistí en mis enseñanzas 0 consejos ante mis colegas en lo que decía el filósofo Francis Bacon.

El estudio y la sabiduría no son fines en sí mismos, ya que un conocimiento sin aplicación concreta es una triste vanidad académica. Yo he ampliado el concepto agregándole que: El conocimiento sin responsabilidad social podría ser tan estéril como el servicio a la comunidad sin una buena preparación académica. $Y$ finalmente, que debemos tener siempre presente el carácter no absoluto del saber, fundamento de la única y verdadera ciencia incluyendo la medicina.

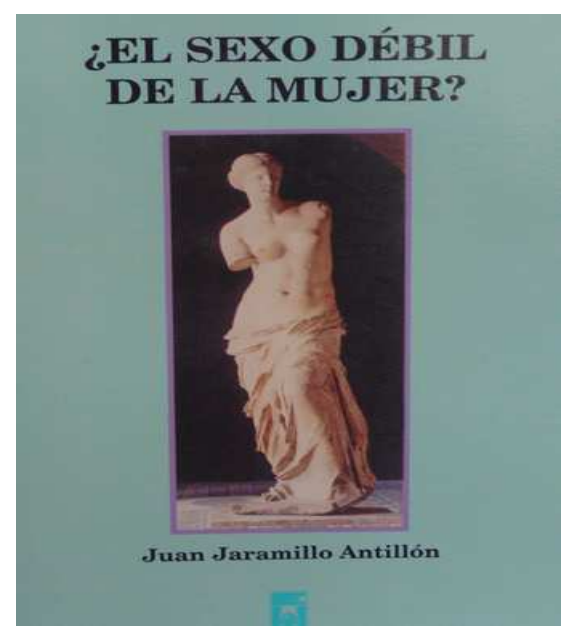

Revista electrónica publicada por el Departamento de Farmacología de la Escuela de Medicina de la Universidad de Costa Rica, 2060 San José, Costa Rica. ${ }^{\circledR}$ All rights reserved. 